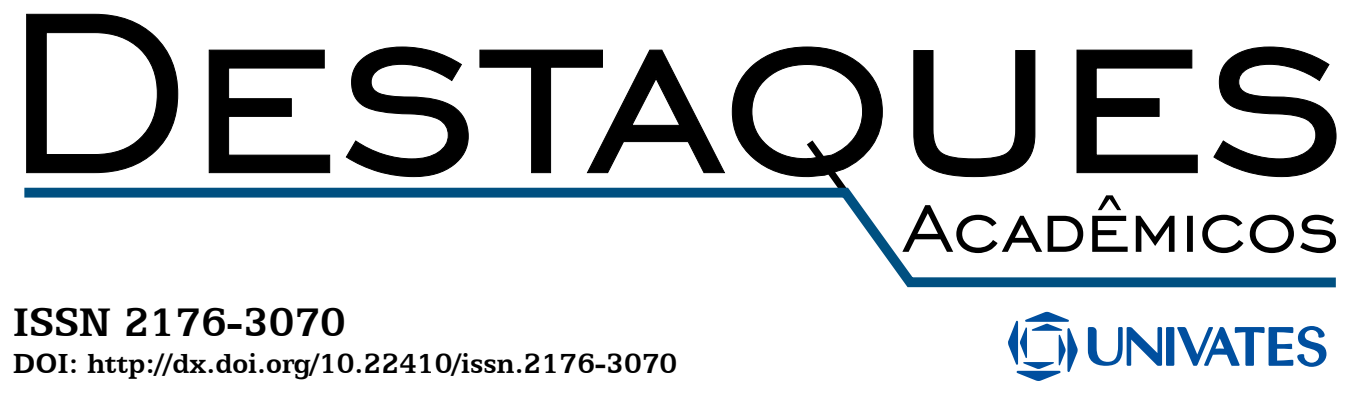

\title{
CIÊNCIAS HUMANAS E SOCIAIS
}

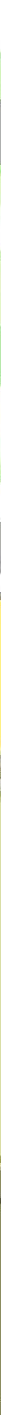




\section{VIIIIUNIVATES}

Universidade do Vale do Taquari - Univates

Reitor: Prof. Me. Ney José Lazzari

Vice-Reitor e Presidente da Fuvates: Prof. Dr. Carlos Cândido da Silva Cyrne

Pró-Reitora de Pesquisa, Extensão e Pós-Graduação: Profa. Dra. Maria Madalena Dullius

Pró-Reitora de Ensino: Profa. Dra. Fernanda Storck Pinheiro

Pró-Reitora de Desenvolvimento Institucional: Profa. Dra. Júlia Elisabete Barden

Pró-Reitor Administrativo: Prof. Me. Oto Roberto Moerschbaecher

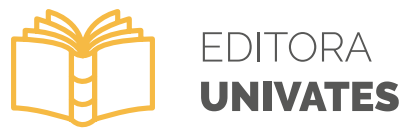

\section{Editora Univates}

Coordenação: Ana Paula Lisboa Monteiro

Editoração e capa: Glauber Röhrig e Marlon Alceu Cristófoli

\section{Conselho Editorial da Editora Univates}

\section{Titulares}

Alexandre André Feil

André Anjos da Silva

Fernanda Rocha da Trindade

João Miguel Back

Sônia Elisa Marchi Gonzatti

\author{
Suplentes \\ Fernanda Cristina Wiebusch Sindelar \\ Claudete Rempel \\ Adriane Pozzobon \\ Rogério José Schuck \\ Evandro Franzen
}

Avelino Tallini, 171 - Bairro Universitário - Lajeado - RS, Brasil Fone: (51) 3714-7024 / Fone: (51) 3714-7000, R.: 5984

editora@univates.br / http://www.univates.br/editora

D476 Destaques Acadêmicos / Centro Universitário Univates - Vol. 1, no. 1 (2009) - Lajeado, RS: Ed. da Univates, 2020.

v. 12, n. 2, 2020.

Instituição mudou o nome para Universidade do Vale do Taquari - Univates em 2017.

Trimestral

ISSN 2176-3070

1. Ensino. 2. Ensino multidisciplinar. I. Universidade do Vale do Taquari - Univates.

CDU: 378

Catalogação na publicação - Biblioteca Univates

Bibliotecária Andrieli Mara Lanferdini - CRB 10/2279

Os artigos são de exclusiva responsabilidade do(s) autor(es).

(C) Fundação Vale do Taquari de Educação e Desenvolvimento Social - FUVATES 


\section{DESTAOUES}

Conselho Editorial da Revista

Cristiane Antonia Hauschild

João Miguel Back

Magali Teresinha Quevedo Grave

Marlon Dalmoro

\section{Coordenador desta edição}

João Miguel Back

Pareceristas desta edição

Alessandro Menna Alves

Alice Krämer Iorra Schmidt

Derli Juliano Neuenfeldt

Elisabete Cristina Barreto Muller

Fabiane Olegário

João Miguel Back

Leila Viviane Scherer Hammes

Magali Quevedo Grave

Marcelo Leandro Dos Santos

Márcio Geller Marques

Maria Elisabete Bersch

Mateus Dalmaz

Sandro Fröhlich

Sérgio Nunes Lopes

Tania Micheline Miorando

Tatiele Gisch Kuntz

Thaís Carnieletto Müller

Tiago Weizenmann 


\section{APRESENTAÇÃO}

A Revista Destaques Acadêmicos, edição 2020, é composta por estudos na Área de Ciências Humanas e Sociais. Dedica-se a discussões de temas em diversas áreas de saber, como Direito, Psicologia, Filosofia, Educação, História e outros. Esta edição traz abordagens de temas provocativos, atuais e inovadores, levando o leitor a viajar por problemas do dia a dia, questões práticas e inovadoras. As reflexões reforçam o espírito investigativo na academia através da troca de saberes e abordagens transversais, buscando sempre aprofundar os estudos e trazer à mesa discussões sobre temas importantes da comunidade acadêmica e comunidade em geral. Trazer a público as pesquisas e discussões do momento reforça a vocação da Academia. A disposição em publicar e compartilhar conhecimentos demostra a responsabilidade dos profissionais em contribuir para a sua qualificação permanente e responder as demandas da sociedade. A UNIVATES tem demostrado, ao longo do tempo, seu empenho no fomento de pesquisas e trocas de conhecimentos entre seus docentes e discentes junto a pesquisadores de outras Instituições. A publicidade e cooperação na construção do conhecimento é uma das formas mais efetivas de qualificar os processos democráticos e promover a cidadania ampla e irrestrita, cumprindo com o papel essencial da Academia de Ensino.

Boa leitura a todos.

Prof. Dr. João Miguel Back

Coordenador desta edição 


\section{SUMÁRIO}

QUEM SÃO OS/AS CONDUTORES/AS COM CNH NO RIO GRANDE

DO SUL? QUESTÕES PARA A PSICOLOGIA DO TRÂNSITO. .7

Bárbara Bernardy, Priscila Pavan Detoni

COMPORTAMENTO DE RISCO E A CONTRIBUIÇÃO DA

PSICOLOGIA PARA A REDUÇÃO DOS ACIDENTES DE TRÂNSITO:

UMA REVISÃO DA LITERATURA.

Neusa Maria Zanon, Luciane De Fátima Rotth Brisotto

A EVOLUÇÃO HISTÓRICA DO ESTADO E O DIREITO

INTERNACIONAL: COORDENAÇÃO OU SUBORDINAÇÃO? 42

Alana Petry

DANOS MORAIS INDENIZÁVEIS DECORRENTES DO ABALO

PSICOLÓGICO NO AMBIENTE DE TRABALHO: CHEFE NARCISISTA

DESTRUTIVO X EMPREGADO.

Monica Alves Rademann, Rafael Zago da Silva

METODOLOGIAS ATIVAS NO PROCESSO DE ENSINO E APRENDIZAGEM NA EDUCAÇÃO PROFISSIONAL. .79

Horácio Nascimento Saccól, Edson Moacir Ahlert

OS DIREITOS FUNDAMENTAIS NO ORDENAMENTO JURÍDICO BRASILEIRO .94

Douglas Luciano de Oliveira

ANÁLISE DA FORMAÇÃO CONTINUADA E PERMANENTE DOS PROFISSIONAIS DE ENFERMAGEM DE NÍVEL TÉCNICO DA ATENÇÃO BÁSICA DE UM MUNICÍPIO DO VALE DO TAQUARI/RS.....101

Carina Guidoni, Edson Moacir Ahlert

METODOLOGIAS ATIVAS NOS CURSOS TÉCNICOS DE ADMINISTRAÇÃO: REVISÃO INTEGRATIVA 116

Luana De Maman, Maria Claudete Schorr

A AFETIVIDADE NOS ANOS INICIAIS DA EDUCAÇÃO BÁSICA:

REVISÃO DE LITERATURA 128

Gilma Helena Souza, Thatiany De Paula, Marcos Venicio Esper 
Antonio Gomes da Costa Neto

EDUCAÇÃO DO CAMPO: A ESCOLA MUNICIPAL BOM JESUS NA PERCEPÇÃO DOS ATORES SOCIAIS ENVOLVIDOS

Edilaine Ferreira Leal, Marcelo Franco Leão

OS SINDICATOS NO BRASIL SOB A LUZ DA TEORIA DA “LÓGICA

DA AÇÃO COLETIVA" DE MANCUR OLSON. .168

Leonardo Rodrigues Ferreira, Sandberg Marcel Santos

EDUCAÇÃO FÍSICA NO ENSINO MÉDIO: DIÁLOGOS COM

ESTUDANTES SOBRE O DESINTERESSE PELAS EXPERIMENTAÇÕES

CORPORAIS

Wellington Kupka Claus, Leandro Oliveira Rocha

INFLUÊNCIA DA FAMÍLIA NA APRENDIZAGEM DE ESTUDANTES

DE UMA ESCOLA PRIVADA MATO-GROSSENSE

Isabel Aparecida Teixeira, Ana Claudia Tasinaffo Alves,

Marcelo Franco Leão

MILITARIZAÇÃO DA ESCOLA PÚBLICA: UMA REFLEXÃO HISTÓRICA DO SISTEMA EDUCACIONAL BRASILEIRO E DAS LUTAS EM MATO GROSSO .206

Monica Strege Medici, Domenico dos Santos Medici, Marcelo Franco Leão 